\section{Evaluación nutricional de escolares en una ciudad fronteriza entre Estados Unidos y México}

\author{
MARIEL A. AVIÑA-BARRERA ${ }^{1, \mathrm{a}}$, OCTELINA CASTILLO-RUIZ ${ }^{1, \mathrm{~b}}$, \\ FRANCISCO VÁZQUEZ-NAVA ${ }^{2, \mathrm{c}}$, ADRIANA PERALES-TORRES $^{1, \mathrm{~d}}$, \\ SANJUANA ALEMAN-CASTILLO ${ }^{1, \mathrm{e}}$
}

\section{Nutritional status of Mexican school age children, living in the frontier with United States}

Background: Undernutrition and obesity coexist among Mexican children due to poverty, sedentariness and inadequate food intake. Aim: To assess the nutritional status of school age children in a Mexican city located in the frontier with United States. Material and Methods: Cross sectional assessment of children from 28 basic schools in 2005, 2008 and 2013. Using a cluster sampling methodology, 5 children per course were selected in each school, reaching a final sample 840 children aged 7 to 12 years old. Body mass index $z$ scores were calculated. Results: The prevalence of overweight and obesity among these children was 49, 54 and 45\% in the assessments performed in 2005, 2008 and 2013 respectively. Conclusions: There is a trend towards a decrease in the frequency of obesity in these children from 2005 to 2013.

(Rev Med Chile 2016; 144: 347-354)

Key words: Child; Nutrition Assessment; Pediatric Obesity.

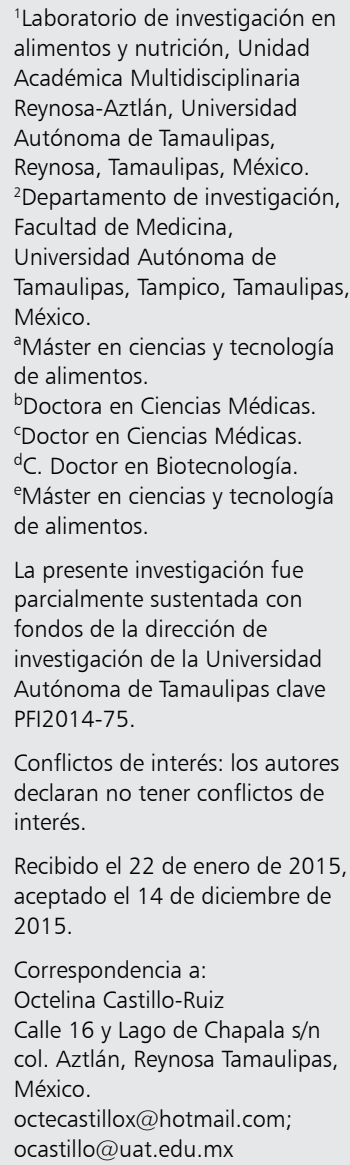

Recibido el 22 de enero de 2015 , aceptado el 14 de diciembre de 2015.

Correspondencia a:

Octelina Castillo-Ruiz

Calle 16 y Lago de Chapala s/n col. Aztlán, Reynosa Tamaulipas, México.

octecastillox@hotmail.com ocastillo@uat.edu.mx

\section{L} os niños en edad escolar necesitan una alimentación adecuada con el objetivo de un sano desarrollo, evitar enfermedades y estar físicamente activos para evitar una sobre nutrición o desnutrición ${ }^{1}$. La desnutrición es un factor de riesgo para el desarrollo infantil, produciendo lesiones a nivel psicomotor que dan como resultado bajo rendimiento académico y una menor capacidad productiva ${ }^{2}$ ocasionada por ingestión dietética inadecuada, infecciones y pobreza ${ }^{3}$, por su parte, el sobrepeso y obesidad dan paso a otras enfermedades de carácter crónico como diabetes mellitus, hipertensión arterial, dislipidemias y enfermedad renal crónica ${ }^{4}$ como resultado de una dieta con mayor densidad energética, disminución en la ingesta de carbohidratos complejos, frutas, verduras y fibra ${ }^{5,6}$, así como la constante migración, el ver televisión y baja actividad física ${ }^{7}$ entre otros. Es un problema que también afecta al estado emocional de los niños, ya que frecuentemente son víctimas de rechazo en la escuela, lo que provoca sentimientos de inferioridad y depresión. Además, se ha documentado que el sobrepeso en la edad infantil es un predictor para el desarrollo de obesidad en la vida adulta ${ }^{8}$.

Dichos problemas nutricionales coexisten en la región fronteriza entre México-Estados Unidos de América, que se extiende a lo largo de 3.141 km desde el Golfo de México al Océano Pacífico. Incluye 94 municipios en 6 estados mexicanos y 48 condados en 4 estados estadounidenses ${ }^{9}$, de ellos, el estado de Texas (EUA) colinda con Tamaulipas (México) que según datos del Instituto Nacional de Estadística, Geografía e Informática (INEGI), cuenta con una población de 3.268.554 habitantes de los cuales 608.891 corresponden al 
municipio de Reynosa ${ }^{10}$. Debido a la transferencia de población en la región de la frontera, la salud de los texanos que viven en esta área requiere intervenciones y estrategias basadas en la colaboración binacional. Sin embargo, cada país tiene sus propias políticas y prácticas de salud ${ }^{11}$.

En México se han realizado algunos esfuerzos por implementar programas nutricionales basados en la educación y promoción para la salud tales como el programa de Acción Específico 2007-2012 Escuela y Salud, Oportunidades, y el Acuerdo Nacional para la Salud Alimentaria ${ }^{12}$ destacando la necesidad de educar en alimentación saludable a los niños desde la educación pre básica considerando estrategias que involucren a niños, sus padres y profesores ${ }^{13}$.

El objetivo de esta investigación fue evaluar el estado nutricio en los escolares de una ciudad fronteriza mexicana en los años 2005, 2008 y 2013, lo cual permite evaluar, de manera indirecta, la efectividad de los esfuerzos en políticas de salud alimentaria.

\section{Material y Métodos}

El presente estudio se realizó en Reynosa, Tamaulipas que contaba en los años 2005 a 2013 con una población escolar de educación básica de 140.000 a 142.000 niños por ciclo escolar'.

Es un estudio transversal comparativo integrado por escolares de 7 a 12 años seleccionados en tres diferentes tiempos: 2005, 2008 y 2013 y evaluados en los meses de septiembre-noviembre. Se realizó un muestreo por conglomerados bietápico, para la selección de las primarias y de los niños de cada grado escolar. En la primera etapa se seleccionaron 28 primarias ubicadas en diferentes sectores y zonas escolares de 119 registradas en la lista oficial de primarias -turno matutino- de la secretaría de educación pública (SEP). En la segunda etapa se seleccionaron aleatoriamente 5 niños de cada grado escolar (primero a sexto grado) con técnica de sustitución en caso de que alguno no aceptara participar en el estudio y exclusión de alumnos con alguna discapacidad que pudiera alterar su estado nutricio. En los tres cortes de tiempo se utilizaron iguales métodos de selección y exclusión de muestra, así como la autorización para las mediciones corporales tanto de las instituciones seleccionadas, como del niño y del padre o tutor por medio de una firma de consentimiento informado, aproba- do por el comité de ética de la Unidad Académica Multidisciplinaria Reynosa-Aztlán perteneciente a la Universidad Autónoma de Tamaulipas.

La recopilación de los datos se realizó mediante entrevistas con los padres que autorizaron la evaluación y, para las mediciones antropométricas de los escolares, se aplicaron técnicas reportadas por la Norma Oficial Mexicana NOM-031-SSA2-1999, titulada atención a la salud del niño ${ }^{14}$ y la Norma Oficial Mexicana NOM-043-SSA2-2012, de Servicios básicos de salud, Promoción y educación para la salud en materia alimentaria y Criterios para brindar orientación ${ }^{15}$.

El material utilizado fue una báscula marca Tanita modelo BF-689 para el peso corporal; un estadiómetro SECA 213 para la estatura y una cinta de fibra de vidrio marca SECA 200 para circunferencia de cintura.

Para diagnosticar el estado nutricio se utilizó el puntaje Z del índice de masa corporal (IMC). Clasificándose con los puntos de corte de la Organización Mundial de la Salud (OMS) para 5 a 19 años de edad consideradas como bajo peso $(\leq-2 \mathrm{DE})$; peso normal $(-1,99 \mathrm{DE} \leq 1 \mathrm{DE})$; sobrepeso ( $>1 \mathrm{DE} \leq 2 \mathrm{DE})$; obesidad ( $>2 \mathrm{DE})$.

\section{Análisis estadístico}

Se calculó el puntaje Z con el programa WHO Anthro Plus v1.0.4, 2007 y para el procesamiento de datos se utilizó la versión 7 del software Statistica. Los datos se analizaron utilizando estadística descriptiva y la prueba factorial ANOVA seleccionando como variable dependiente el puntaje Z-IMC y como factores categóricos, el año y género, con el fin de identificar si existían diferencias en el estado nutricional entre los tres años evaluados (2005, 2008 y 2013) y una prueba post hoc de Bonferroni para observar en que grupos se presentaron diferencias, considerando como estadísticamente significativo un valor $\mathrm{p}<0,05$. Los resultados obtenidos en gráficas o tablas se expresan en números y porcentajes.

\section{Resultados}

Se evaluaron 840 alumnos por año sumando una muestra total de 2.520 escolares en los tres años, su distribución por edad y género se muestra en la Tabla 1, donde se observa la mayor proporción de población constituida por niñas; $51 \%$ (2005), 51,8\% (2008) y 52,4\% (2013). 
Tabla 1. Descripción de la muestra de escolares por edad y género en los tres años evaluados

\begin{tabular}{|c|c|c|c|}
\hline & $\begin{array}{c}2005 \\
\text { n } \quad(\%)\end{array}$ & $\begin{array}{c}2008 \\
\text { n } \quad(\%)\end{array}$ & $\begin{array}{c}2013 \\
\text { n } \quad(\%)\end{array}$ \\
\hline \multicolumn{4}{|c|}{ Niñas } \\
\hline 7 & $83(55,3)$ & $77(52,7)$ & $76(55,1)$ \\
\hline 8 & $69(49,3)$ & $83(45,4)$ & $94(60,6)$ \\
\hline 9 & $63(47,4)$ & $85(55,6)$ & $70(49,3)$ \\
\hline 10 & $69(50)$ & $73(50)$ & $82(53,2)$ \\
\hline 11 & $74(52,9)$ & $68(57,6)$ & $74(48,1)$ \\
\hline 12 & $71(51,1)$ & $49(52,1)$ & $44(45,4)$ \\
\hline Total & $429(51,07)$ & $435(51,8)$ & $440(52,4)$ \\
\hline \multicolumn{4}{|c|}{ Niños } \\
\hline 7 & $67(44,7)$ & $69(47,3)$ & $62(44,9)$ \\
\hline 8 & $71(50,7)$ & $100(54,6)$ & $61(39,4)$ \\
\hline 9 & $70(52,6)$ & $68(44,4)$ & $72(50,7)$ \\
\hline 10 & $69(50)$ & $73(50)$ & $72(46,8)$ \\
\hline 11 & $66(47,1)$ & $50(42,4)$ & $80(51,9)$ \\
\hline 12 & $68(48,9)$ & $45(47,9)$ & $53(54,6)$ \\
\hline Total & $411(48,93)$ & $405(48,2)$ & $400(47,6)$ \\
\hline
\end{tabular}

\section{Estado nutricional}

La prevalencia de sobrepeso fue de $22,4 \%$, $19,5 \%$ y $20,6 \%$ para los años 2005, 2008 y 2013 respectivamente sin diferencias significativas entre grupos (Tabla 2). Para el caso de obesidad fueron de $27,1 \%, 34,4 \%$ y $24,8 \%$ respectivamente con diferencias significativas determinadas por medio del post hoc de Bonferroni (2005 vs 2008, $\mathrm{p}=0,000 ; 2005$ vs $2013, \mathrm{p}=0,001 ; 2008$ vs 2013 , $\mathrm{p}=0,000)$ mientras que el bajo peso presentó mayor prevalencia en el año 2013 en comparación con el $2005(\mathrm{p}=0,000)$.

Se analizaron (ANOVA y post hoc de Bonferroni) las medidas corporales, encontrándose diferencias estadísticamente significativas en ambos géneros en cuanto al peso: en el año 2008 vs 2013 en niñas $(p=0,008)$ y niños $(p=0,014)$ de 7 años, además en este último género, también se encontraron diferencias en el año 2005 vs

Tabla 2. Estado Nutricional por medio del Puntaje Z de IMC por género en escolares

\begin{tabular}{|c|c|c|c|}
\hline & Niños & Niñas & Total \\
\hline $\begin{array}{l}2005 \\
\text { Bajo peso } \\
(\leq-2 \mathrm{DE})\end{array}$ & $\begin{array}{l}(n=411) \\
1(0,24 \%) \\
0,23-0,72\end{array}$ & $\begin{array}{l}(n=429) \\
9(2,09 \%) \\
0,74-3,45\end{array}$ & $\begin{array}{c}(\mathbf{n}=\mathbf{8 4 0}) \\
10(1,19 \%) \\
0,5-1,9\end{array}$ \\
\hline $\begin{array}{l}\text { Normal } \\
(-1,99 \mathrm{DE}+1 \mathrm{DE})\end{array}$ & $\begin{array}{c}206(50,12 \%) \\
45,3-54,9\end{array}$ & $\begin{array}{c}200(46,62 \%) \\
41,9-51,3\end{array}$ & $\begin{array}{c}406(48,33 \%) \\
44,95-51,7\end{array}$ \\
\hline $\begin{array}{l}\text { Sobrepeso } \\
(>+1 \mathrm{DE} \leq+2 \mathrm{DE})\end{array}$ & $\begin{array}{c}73(17,76 \%) \\
14,1-21,4\end{array}$ & $\begin{array}{c}115(26,8 \%) \\
22,6-30,9\end{array}$ & $\begin{array}{c}188(22,38 \%) \\
19,6-25,2\end{array}$ \\
\hline $\begin{array}{l}\text { Obesidad } \\
(>+2 \mathrm{DE})\end{array}$ & $\begin{array}{c}131(31,87 \%) \\
27,4-36,37\end{array}$ & $\begin{array}{c}105(24,47 \%) \\
20,4-28,5\end{array}$ & $\begin{array}{c}236(27,09 \%) \\
23,7-31,1\end{array}$ \\
\hline $\begin{array}{l}2008 \\
\text { Bajo peso } \\
(\leq-2 \mathrm{DE})\end{array}$ & $\begin{array}{c}(n=405) \\
10(2,47) \\
0,96-3,98\end{array}$ & $\begin{array}{c}(n=435) \\
10(2,3 \%) \\
0,89-3,7\end{array}$ & $\begin{array}{c}(\mathbf{n}=\mathbf{8 4 0}) \\
20(2,38 \%) \\
1,35-3,4\end{array}$ \\
\hline $\begin{array}{l}\text { Normal } \\
(-1,99 \mathrm{DE}+1 \mathrm{DE})\end{array}$ & $\begin{array}{c}168(41,48 \%) \\
36,7-46,3\end{array}$ & $\begin{array}{c}199(45,75 \%) \\
41,1-50,4\end{array}$ & $\begin{array}{c}367(43,69 \%) \\
40,3-47\end{array}$ \\
\hline $\begin{array}{l}\text { Sobrepeso } \\
(>+1 \mathrm{DE} \leq+2 \mathrm{DE})\end{array}$ & $\begin{array}{c}60(14,81 \%) \\
11,4-18,2\end{array}$ & $\begin{array}{c}104(23,91 \%) \\
19,9-27,9\end{array}$ & $\begin{array}{c}164(19,52 \%) \\
16,8-22,2\end{array}$ \\
\hline $\begin{array}{l}\text { Obesidad } \\
(>+2 \mathrm{DE})\end{array}$ & $\begin{array}{c}167(41,23 \%) \\
36,4-46\end{array}$ & $\begin{array}{c}122(28,05 \%) \\
23,8-32,3\end{array}$ & $\begin{array}{c}289(34,4 \%) \\
31,2-37,6\end{array}$ \\
\hline $\begin{array}{l}2013 \\
\text { Bajo peso } \\
(\leq-2 \mathrm{DE})\end{array}$ & $\begin{array}{c}(\mathbf{n}=\mathbf{4 0 0 )}) \\
22(5,5 \%) \\
3,3-7,7\end{array}$ & $\begin{array}{c}(n=440) \\
12(2,73 \%) \\
1,2-4,25\end{array}$ & $\begin{array}{c}(n=840) \\
34(4,05 \%) \\
2,7-5,4\end{array}$ \\
\hline $\begin{array}{l}\text { Normal } \\
(-1,99 \mathrm{DE}+1 \mathrm{DE})\end{array}$ & $\begin{array}{c}187(46,75 \%) \\
41,9-51,6\end{array}$ & $\begin{array}{c}238(54,09 \%) \\
49,4-58,7\end{array}$ & $\begin{array}{c}425(50,59 \%) \\
47,2-53,9\end{array}$ \\
\hline $\begin{array}{l}\text { Sobrepeso } \\
(>+1 \mathrm{DE} \leq+2 \mathrm{DE})\end{array}$ & $\begin{array}{c}78(19,5 \%) \\
15,6-23,4\end{array}$ & $\begin{array}{c}95(21,59 \%) \\
17,7-25,4\end{array}$ & $\begin{array}{c}173(20,60 \%) \\
17,8-24,5\end{array}$ \\
\hline $\begin{array}{l}\text { Obesidad } \\
(>+2 \mathrm{DE})\end{array}$ & $\begin{array}{c}113(28,25 \%) \\
23,8-32,6\end{array}$ & $\begin{array}{c}95(21,59 \%) \\
17,7-25,4\end{array}$ & $\begin{array}{c}208(24,76 \%) \\
20,6-27,7\end{array}$ \\
\hline
\end{tabular}

Nota: Los datos se muestran con el IC 95\%. 
Evaluación nutricional en escolares de ciudad fronteriza - M. A. Aviña-Barrera et al

$2008(\mathrm{p}=0,015)$. Para el grupo de 8 años fueron significativos 2008 vs 2013 para niñas y niños ( $\mathrm{p}=0,027 ; 0,014$ respectivamente); los varones de 11 años presentaron cambios significativos entre el año 2008 vs 2005 ( $\mathrm{p}=0,002)$ y 2008 vs 2013 $(\mathrm{p}=0,002)$, para el caso de la estatura se presentan diferencias en las niñas de 10 años (2005 vs 2008: $\mathrm{p}=0,014)$ y en los niños de 11 (2008 vs 2005: $\mathrm{p}=0,007$ y 2008 vs 2013: $\mathrm{p}=0,029)$ (Tabla 3).
Con respecto al IMC-puntaje $\mathrm{z}$ se observaron diferencias significativas en el año 2008 vs 2013 a los 7 y 8 años de edad tanto para las niñas ( $\mathrm{p}=0,008$; 0,037 respectivamente) como para los varones $(\mathrm{p}=0,027 ; 0,001)$, a la edad de 11 años los niños presentaron diferencias entre el año 2008 comparado con 2005 y 2013 ( $\mathrm{p}=0,030 ; 0,027)$ así como en la circunferencia de cintura del año 2005 vs $2008(\mathrm{p}=0,013)$ como se muestra en la Tabla 4.

Tabla 3. Media y DE de variables antropométricas estudiadas (peso y estatura) por edad, género y año de evaluación

\begin{tabular}{|c|c|c|c|c|c|c|c|c|}
\hline & 2005 & $\begin{array}{l}\text { Peso } \\
2008\end{array}$ & 2013 & Valor p & 2005 & $\begin{array}{c}\text { Estatura } \\
2008\end{array}$ & 2013 & Valor p \\
\hline \multicolumn{9}{|c|}{ Niñas } \\
\hline 7 & $26,7 \pm 7,47$ & $28,7 \pm 7,54$ & $25,3 \pm 5,84$ & $0,010^{*}$ & $122,5 \pm 6,09$ & $124,4 \pm 7,33$ & $122,8 \pm 6,93$ & 0,182 \\
\hline 8 & $31,2 \pm 8,46$ & $32,8 \pm 10,57$ & $29,2 \pm 7,93$ & $0,032 *$ & $129,5 \pm 6,07$ & $129,6 \pm 7,13$ & $128,1 \pm 6,85$ & 0,282 \\
\hline 9 & $38,3 \pm 12,36$ & $37,3 \pm 14,78$ & $36,5 \pm 11,24$ & 0,744 & $136,6 \pm 8,55$ & $136,2 \pm 8,19$ & $135,7 \pm 8,01$ & 0,821 \\
\hline 10 & $43,9 \pm 11,72$ & $40,7 \pm 13,88$ & $40,2 \pm 8,75$ & 0,144 & $143,3 \pm 7,47$ & $139,4 \pm 8,47$ & $141,2 \pm 7,86$ & $0,018^{*}$ \\
\hline 11 & $45,5 \pm 11,37$ & $47,3 \pm 12,49$ & $45,6 \pm 12,98$ & 0,625 & $147 \pm 7,39$ & $148 \pm 8,35$ & $147,6 \pm 5,85$ & 0,698 \\
\hline 12 & $50,3 \pm 12,48$ & $54,3 \pm 15,13$ & $51,6 \pm 13,21$ & 0,295 & $152,2 \pm 5,93$ & $150,6 \pm 8,11$ & $153,2 \pm 6,84$ & 0,201 \\
\hline \multicolumn{9}{|c|}{ Niños } \\
\hline 7 & $26,8 \pm 6,87$ & $30,9 \pm 9,86$ & $27,4 \pm 8,18$ & $0,011^{*}$ & $123,3 \pm 6,01$ & $126,2 \pm 9,20$ & $125,6 \pm 6,37$ & 0,061 \\
\hline 8 & $31,5 \pm 9,13$ & $34,3 \pm 11,88$ & $29,5 \pm 9,23$ & $0,014^{*}$ & $129,5 \pm 5,78$ & $129,4 \pm 8,28$ & $129,3 \pm 7,79$ & 0,989 \\
\hline 9 & $36,4 \pm 8,98$ & $38,3 \pm 14,28$ & $34,6 \pm 8,05$ & 0,126 & $136,5 \pm 6,33$ & $135,7 \pm 7,22$ & $136,0 \pm 6,45$ & 0,765 \\
\hline 10 & $43,3 \pm 12,52$ & $41,6 \pm 13,07$ & $40,2 \pm 11,92$ & 0,335 & $141,6 \pm 6,25$ & $139,6 \pm 8,02$ & $142,3 \pm 6,24$ & 0,051 \\
\hline 11 & $44,6 \pm 13,84$ & $53,8 \pm 16,63$ & $44,9 \pm 12,41$ & 0,001 * & $144,7 \pm 8,20$ & $149,5 \pm 9,96$ & $145,5 \pm 7,65$ & $0,006^{*}$ \\
\hline 12 & $51,2 \pm 17,21$ & $47,6 \pm 13,34$ & $52,5 \pm 11,70$ & 0,232 & $150,8 \pm 7,47$ & $150,6 \pm 9,26$ & $153,6 \pm 7,14$ & 0,099 \\
\hline
\end{tabular}

*Diferencia significativa estadísticamente entre los años 2005, 2008 y 2013, por medio de ANOVA p < 0,05.

Tabla 4. Media y DE de variables antropométricas estudiadas (puntaje IMC-puntaje Z y CC) por edad, género y año de evaluación

\begin{tabular}{|c|c|c|c|c|c|c|c|c|}
\hline & \multicolumn{3}{|c|}{ IMC- Puntaje Z } & \multicolumn{5}{|c|}{ Circunferencia de Cintura } \\
\hline & 2005 & 2008 & 2013 & Valor $p$ & 2005 & 2008 & 2013 & Valor $p$ \\
\hline \multicolumn{9}{|c|}{ Niñas } \\
\hline 7 & $0,75 \pm 1,47$ & $1,07 \pm 1,56$ & $0,35 \pm 1,38$ & $0,011 *$ & $59,9 \pm 8,9$ & $62,9 \pm 10,6$ & $60,9 \pm 8,62$ & 0,118 \\
\hline 8 & $0,9 \pm 1,37$ & $1,08 \pm 1,69$ & $0,48 \pm 1,58$ & $0,036^{*}$ & $62,4 \pm 9,17$ & $65 \pm 11,39$ & $62,5 \pm 9,14$ & 0,165 \\
\hline 9 & $1,15 \pm 1,44$ & $0,85 \pm 1,77$ & $0,96 \pm 1,49$ & 0,511 & $69,2 \pm 12,16$ & $67,4 \pm 14,77$ & $67,3 \pm 10,45$ & 0,624 \\
\hline 10 & $1,26 \pm 1,25$ & $0,95 \pm 1,68$ & $1,02 \pm 1,11$ & 0,370 & $68,9 \pm 13,99$ & $69,2 \pm 13,40$ & $69,7 \pm 9,19$ & 0,907 \\
\hline 11 & $0,87 \pm 1,31$ & $0,99 \pm 1,54$ & $0,89 \pm 1,35$ & 0,770 & $70,4 \pm 11,05$ & $72,4 \pm 10,93$ & $72,2 \pm 12,44$ & 0,528 \\
\hline 12 & $1,07 \pm 1,36$ & $1,36 \pm 1,28$ & $0,81 \pm 1,54$ & 0,162 & $71,2 \pm 11,19$ & $74,1 \pm 12,98$ & $75,1 \pm 12,24$ & 0,200 \\
\hline \multicolumn{9}{|c|}{ Niños } \\
\hline 7 & $0,85 \pm 1,61$ & $1,49 \pm 2,32$ & $0,56 \pm 2,04$ & $0,026^{*}$ & $59,6 \pm 8,85$ & $64,9 \pm 12,07$ & $61,6 \pm 11,80$ & $0,017^{*}$ \\
\hline 8 & $1,09 \pm 1,65$ & $1,60 \pm 2,11$ & $1,12 \pm 2,07$ & 0,001 * & $63,4 \pm 10,52$ & $66,5 \pm 12,17$ & $64,4 \pm 10,03$ & 0,175 \\
\hline 9 & $1,19 \pm 1,44$ & $1,39 \pm 2,17$ & $0,92 \pm 1,34$ & 0,257 & $67,7 \pm 11,46$ & $69,8 \pm 14,96$ & $67,4 \pm 9,16$ & 0,445 \\
\hline 10 & $1,51 \pm 1,54$ & $1,33 \pm 1,68$ & $0,86 \pm 1,76$ & 0,062 & $72,6 \pm 13,08$ & $73,5 \pm 14,22$ & $71,1 \pm 11,91$ & 0,534 \\
\hline 11 & $1,11 \pm 1,41$ & $1,80 \pm 1,40$ & $1,13 \pm 1,42$ & $0,015^{*}$ & $69,3 \pm 17,71$ & $77,4 \pm 15,31$ & $75,3 \pm 11,82$ & $0,009 *$ \\
\hline 12 & $1,11 \pm 1,54$ & $0,69 \pm 1,62$ & $1,23 \pm 1,38$ & 0,193 & $74,6 \pm 15,93$ & $73,7 \pm 12,89$ & $77,8 \pm 12,24$ & 0,302 \\
\hline
\end{tabular}

*Diferencia significativa estadísticamente entre los años 2005, 2008 y 2013, por medio de ANOVA p < 0,05. 

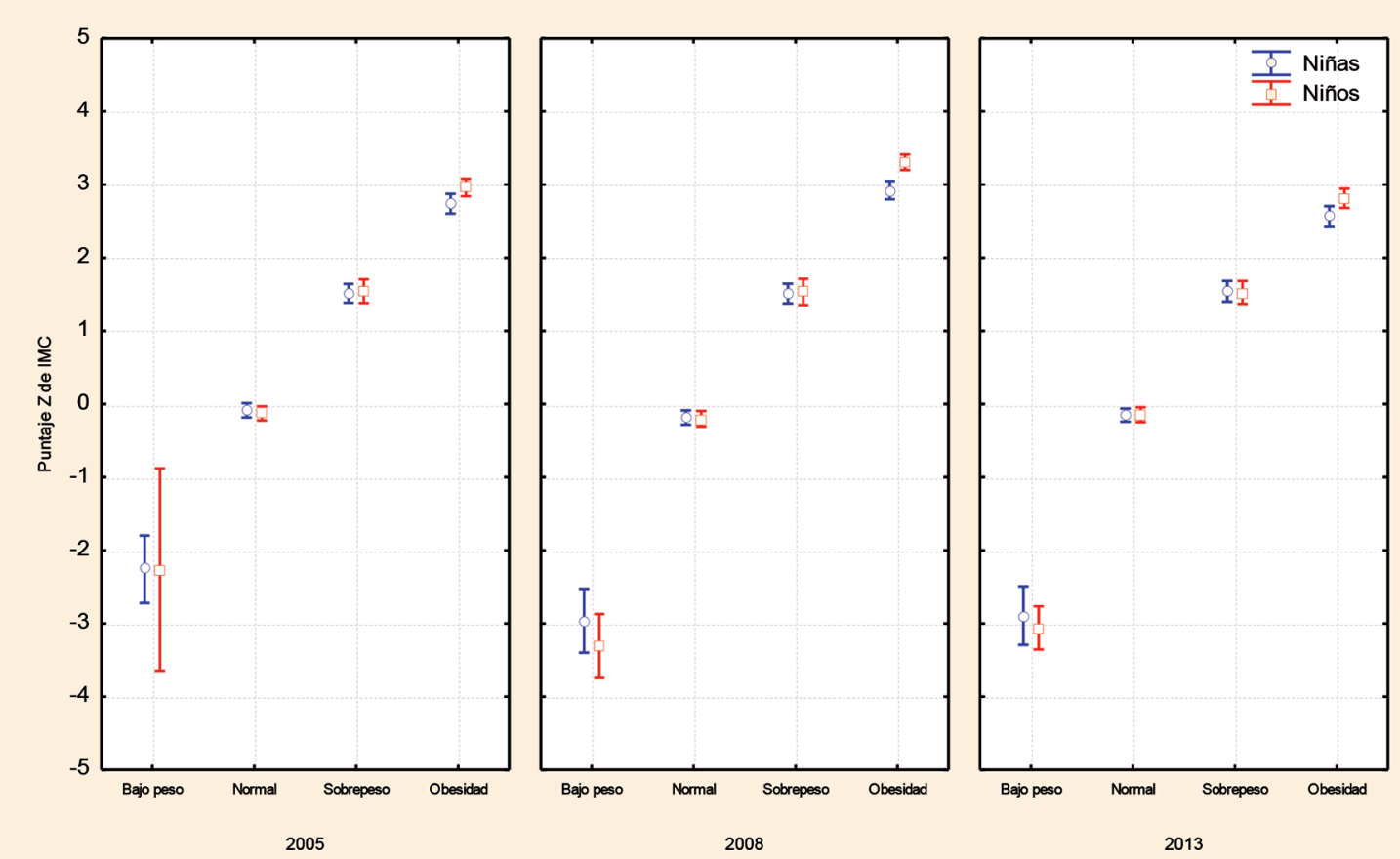

Figura 1. Estado nutricio de los escolares en cada año de evaluación por género.

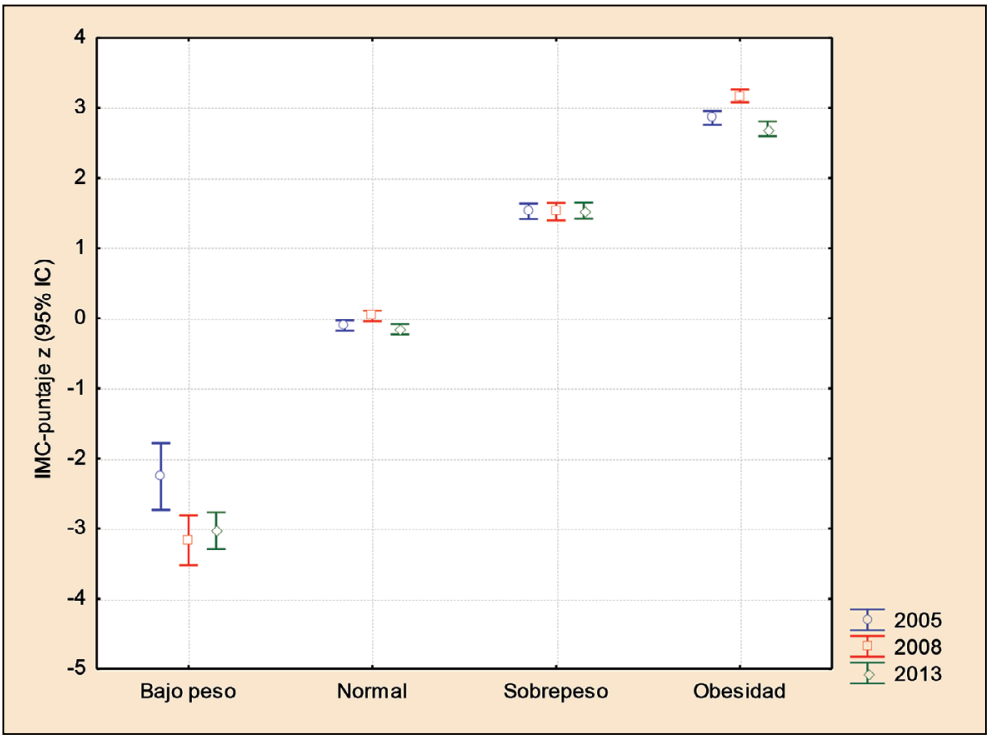

Figura 2. Evaluación nutricional de los escolares de Reynosa, Tamaulipas.

En la Figura 1 se aprecia la media y desviación estándar de cada estado nutricio con un intervalo de confianza del 95\%, los resultados mostrados corresponden a la comparación entre los tres años evaluados por género $(\mathrm{p}=0,039)$, en el
2008 las niñas con obesidad presentaron menor IMC-puntaje $Z$ en comparación a los varones, con diferencia significativa de $\mathrm{p}=0,001$ (post hoc de Bonferroni). Cabe mencionar que los niños con obesidad ese mismo año presentaron diferencias 
en el IMC-puntaje Z, más elevado en comparación a los años 2005 y 2013 de ambos géneros (niños $\mathrm{p}=0,008,0,000$; niñas $\mathrm{p}=0,000,0,000$ respectivamente). Sin embargo, el bajo peso y sobrepeso no presentaron diferencias, como se muestra en la Figura 2.

\section{Discusión}

En nuestro estudio se detectó un descenso de 8,56 puntos porcentuales en sobrepeso/obesidad del año 2008 (53,9\%) al 2013 (45,4\%), sin embargo, sigue siendo una elevada prevalencia comparado con la Encuesta Nacional de Salud y Nutrición Tamaulipas 2012 (ENSANUT Tamaulipas 2012) que presenta una prevalencia de sobrepeso/obesidad de 38,9\% ${ }^{16}$; así mismo datos de la Encuesta Nacional de Salud en Escolares, 2008 mencionan que en Tamaulipas 40 de cada 100 escolares de género masculino presentan este estado nutricional ${ }^{17}$, cifra menor a la observada en la ciudad de Reynosa para el mismo año en el presente estudio.

En el Noroeste de México (Tijuana, Baja California) frontera con California-EUA, se reportó asociación significativa de los niños que cruzaban frecuentemente a EUA con la obesidad (36\%), dicha prevalencia fue mayor a la nacional ${ }^{18} \mathrm{y} \mathrm{a}$ la reportada en el presente estudio en los 3 años de evaluación, consecuentemente los escolares fronterizos se encuentran en un punto crítico de atención para evitar que esta prevalencia continúe o incremente.

En otro estudio realizado en el Noreste de México (Matamoros, Tamaulipas) frontera con Brownsville, Texas (EUA) se reportó mayor porcentaje de adolescentes en frontera del lado americano con riesgo de sobrepeso $(17,2 \%)$ que estudiantes del lado mexicano $(14,8 \%)$ y el porcentaje que ya tenía sobrepeso fue igual en ambos lados $(16,9 \%)^{19}$ es por ello que la atención en edad escolar es imprescindible para que las prevalencias en edades posteriores sean menores. Vilchis-Gil y colaboradores por su parte, encontraron que la diferencia del estado nutricio entre niños con obesidad y eutróficos radica en los malos hábitos de los primeros, como omitir el desayuno, comprar comida en la escuela, y el consumo de alimentos grasos y bebidas azucaradas ${ }^{20}$. Mientras que en EUA, Ogden y colaboradores encontraron una prevalencia de obesidad de 34,2\% en escolares en los años 2011-2012 21 Para el caso de México, fue de $34,4 \%$ según la ENSANUT 201222, cifra similar a la de EUA con quien se comparte frontera.

A partir de la problemática nutricional, en México algunas Instituciones crearon diversos programas y políticas de intervención a nivel nacional, estatal y municipal dirigidos, en un principio, a contrarrestar el hambre (Programa Oportunidades y Desayunos escolares) ${ }^{23}$. Sin embargo, en México se ha observado un importante cambio en el estado nutricional caracterizado por el aumento en el consumo de alimentos con bajo valor nutritivo, comidas "rápidas" y productos alimenticios estadounidenses de fácil importación, altamente calóricos y de frecuente adquisición por escolares ${ }^{24}$ observándose la coexistencia del binomio desnutrición-obesidad por lo que se desarrollaron estrategias como el Acuerdo Nacional para la Salud Alimentaria (ANSA) y programas como "Escuela y Salud"12. Debido a que México continua ocupando uno de los primeros lugares en la prevalencia del sobrepeso/obesidad a nivel mundial, se puso en marcha desde el año 2013, una de las estrategias gubernamentales más amplias para hacerle frente a dicha problemática que incluye la sensibilización y el uso de medidas fiscales y regulatorias ${ }^{25}$.

En conclusión el presente estudio, a través de las mediciones corporales, mostró cambios en el estado nutricional manifestados del año 2005 al 2008 en el aumento de la prevalencia sobrepeso/ obesidad. Sin embargo, a partir del año 2008 al 2013 se refleja un descenso que, de manera indirecta, se pudiera relacionar con la efectividad de estrategias y programas implementados a partir de 2010 en el país dirigidos a contrarrestar la sobrealimentación. No obstante, se recomienda hacer un estudio de carácter directo hacia los programas implementados usando indicadores que evalúen su impacto.

Las limitaciones del estudio son: ser transversal, con evaluaciones realizadas en tres tiempos diferentes lo que impide valorar el cambio nutricional en la misma población; por costos económicos de seguimiento no fue posible realizar un estudio longitudinal.

Este estudio aporta información sobre los cambios en el estado nutricional en una ciudad fronteriza entre EUA y México que, comparado a nivel nacional e internacional, mediante la preva- 
lencia de sobrepeso/obesidad refleja que se deben incrementar los esfuerzos en materia de seguridad alimentaria y promoción de la salud para detener y/o revertir a nivel escolar la incidencia de malnutrición que conduce a un incremento del gasto total en salud pública.

Agradecimientos: Al equipo de supervisión de antropometría de la Unidad Académica Multidisciplinaria Reynosa-Aztlán de la Universidad Autónoma de Tamaulipas, México y a la Secretaría de Educación Pública del Estado de Tamaulipas.

\section{Referencias}

1. Organización de las Naciones Unidas para la Alimentación y la Agricultura [Internet], Estados Unidos: Alimentación escolar; 2015. Disponible en: http://www.fao. org/school-food/es/ [Consultado el 10 de julio de 2015].

2. Mamani-Ortiz Y, Rojas-Salazar EG, Caero-Suárez RI, Choque-Ontiveros MC. Prevalencia de desnutrición en niños y niñas en edad escolar del municipio de Vinto. Rev Méd-Cient "Luz Vida”, 2013; 4 (1): 36-40.

3. Yunes ZJLM, Barrios RA, Duarte OA. Prevalencia de desnutrición, sobrepeso y obesidad en niños del CENDI. Pediatr Méx 2009; 11 (2): 62-6.

4. Basain VJM, Valdés AMC, Miyar Pieiga E, Pérez MM, Tase PS, Ramos AME. Malnutrición por exceso e hipertensión arterial en niños y adolescentes de un área de salud. Rev Arch Med Camagüey 2015; 19 (3): 220-8.

5. Organización Mundial de la Salud. Dieta, nutrición y prevención de enfermedades crónicas. Informe de una Consulta Mixta de Expertos OMS/FAO. Organización Mundial de la Salud. Ginebra: OMS; 2014. Serie de Informes Técnicos; 916. Disponible en: http://www.fao. org/3/a-ac911s.pdf [Consultado el 7 de junio de 2015].

6. Tojo SR, Leis TR. Situaciones fisiológicas y etapas de la vida. Edad infantil. En: Varela MG, Editores, Libro Blanco de la Nutrición de España. Madrid, España: Fundación Española de la Nutrición; 2013. p. 55-64.

7. López-Barrón RG, Jiménez-Cruz A, Bacardí-Gascón M. Modifiable environmental obesity risk factors among elementary school children in a México-US Border city. Nutr Hosp 2015; 31 (5): 2047-53.

8. Lara-García B, Flores-Peña Y, Alatorre-Esquivel MA, Sosa-Briones R, Cerda-Flores RM. Percepción materna de sobrepeso-obesidad infantil y riesgos de salud en Nuevo Laredo, Tamaulipas, México. Salud pública Méx 2011; 53 (3): 258-63.

9. Frontera de Estados Unidos y México. [Monografía en Internet]. Organización Panamericana de la Salud; 2014.
Disponible en: http://www.paho.org/saludenlasamericas/index.php?option $=$ com_content $\& v i e w=$ article\&i$\mathrm{d}=63$ \&Itemid=63\&lang=es [Consultado el 3 de junio de 2015].

10. México en Cifras [Internet], México: Información Nacional, por entidad Federativa y Municipios; 2010. Disponible en: http://www3.inegi.org.mx/sistemas/ mexicocifras $/ ? \mathrm{e}=28 \&$ mun $=032$ [Consultado el 8 de junio de 2015].

11. Delgado E, Castrucci BC, Fonseca V, Dutton RJ, Berrahou F. Lecciones aprendidas de un estudio binacional para examinar el estado de salud de las mujeres en la región fronteriza de México-Estados Unidos. Prev Chronic Dis 2008; 5 (4). Disponible en: http://198.246.124.22/pcd/issues/2008/oct/pdf/08_0073_ es.pdf [Consultado el 6 de junio de 2015].

12. Macías MAI, Gordillo SLG, Camacho REJ. Hábitos alimentarios de niños en edad escolar y el papel de la educación para la salud. Rev Chil Nutr 2012; 39 (3): 40-3.

13. Vio RF, Salinas CJ, González GCG, Huenchupán CM. Conocimientos y consumo alimentario en escolares, sus padres y profesores: un análisis comparativo. Rev Chil Nutr 2012; 39 (3): 34-9.

14. Norma Oficial Mexicana NOM-031-SSA2-1999, Atención a la salud del niño. Diario Oficial de la Federación (9-02-2001).

15. Norma Oficial Mexicana NOM-043-SSA2-2012, Servicios básicos de salud, Promoción y educación para la salud en materia alimentaria y Criterios para brindar orientación. Diario Oficial de la Federación (22-012013).

16. Rivera-Dommarco J, Cuevas-Nasu L, Shamah-Levy T, Valenzuela-Bravo DG, Ávila-Arcos MA. Encuesta Nacional de Salud y Nutrición 2012. Resultados por entidad federativa, Tamaulipas. INSP 2013; 74-9.

17. Shamah-Levy T. editora. Encuesta Nacional de Salud en Escolares 2008. Cuernavaca (México): Instituto Nacional de Salud Pública; 2010.

18. Menchaca MP, Zonana NA. Obesidad en niños mexicanos de la frontera norte. Cartas al editor. Salud Pública Méx 2006; 48 (1): 1.

19. Pérez A, Reininger BM, Aguirre FMI, Sanderson M, Roberts RE. Physical activity and overweight among adolescents on the Texas-Mexico border. Rev Panam Salud Pública 2006; 19 (4): 244-52.

20. Vilchis-Gil J, Galván-Portillo $M$, Klünder-Klünder $M$, Cruz M, Flores-Huerta, S. Food habits, physical activities and sedentary lifestyles of eutrophic and obese school children: a case-control study. BMC Public Health 2015; 15: 124.

21. Ogden CL, Carroll MD, Kit BK, Flegal KM. Prevalence 
of childhood and adult obesity in the Unites States, 2011-2012. JAMA 2014; 311 (8): 806-14. Disponible en: http://jama.jamanetwork.com/article.aspx?articlei$\mathrm{d}=1832542$ \&resultClick $=3$ [Consultado el 4 de diciembre de 2014].

22. Gutiérrez JP, Rivera-Dommarco J, Shamah-Levy T, Villalpando-Hernández S, Franco A, Cuevas-Nasu L, et al. Encuesta Nacional de Salud y Nutrición 2012. Resultados Nacionales. INSP 2012; 147-67.

23. Barquera-Cervera S, Campos-Nonato I, Rojas R, Rivera J. Obesidad en México: epidemiología y políticas de salud para su control y prevención. Gaceta Médica de México 2010; 146 (6): 397-407.

24. Bacardí-Gascón M, Jiménez-Cruz A. TV Food advertising geared to children in Latin-American countries and Hispanics in the USA: a review. Nutr Hosp 2015; 31 (5): 1928-35.

25. Sassi F, Devaux M, Cecchini M. La obesidad y la economía de la prevención: "Fit not fat". Obesity Update. OECD June 2014. Disponible en: http://www.oecd.org/ health/obesity-update.htm [Consultado el 16 de diciembre de 2014]. 